\title{
MESSAGE-MACRO: Linking an Energy Supply Model with a Macroeconomic Module and Solving it Iteratively
}

Sabine Messner

Integrated Resource Management Ltd., Vienna, Austria

Leo Schrattenholzer

International Institute for Applied Systems Analysis, Laxenburg, Austria

RR-00-10

June 2000

Reprinted from Energy 25 (2000) 267-282.

International Institute for Applied Systems Analysis • Schlossplatz 1 - A-2361 Laxenburg • Austria Tel: (+43 2236) 807 • Fax: (+43 2236) 71313 • E-mail: publications@iiasa.ac.at • Web: www.iiasa.ac.at 
Research Reports, which record research conducted at IISA, are independently reviewed before publication. Views or opinions expressed herein do not necessarily represent those of the Institute, its National Member Organizations, or other organizations supporting the work.

Reprinted from Energy 25 (2000) 267-282.

Copyright (c) 2000, with permission from Elsevier Science.

All rights reserved. No part of this publication may be reproduced or transmitted in any form or by any means, electronic or mechanical, including photocopy, recording, or any information storage or retrieval system, without permission in writing from the copyright holder. 


\title{
MESSAGE-MACRO: linking an energy supply model with a macroeconomic module and solving it iteratively
}

\author{
Sabine Messner, Leo Schrattenholzer * \\ Environmentally Compatible Energy Strategies Project, International Institute for Applied Systems Analysis, \\ Schloßplatz 1, A-2361 Laxenburg, Austria
}

Received 11 December 1998

\begin{abstract}
MESSAGE-MACRO is the result of linking a macroeconomic model with a detailed energy supply model. The purpose of the linkage is to consistently reflect the influence of energy supply costs as calculated by the energy supply model in the optimal mix of production factors included in the macroeconomic model. In this article, we describe an automated link of two independently running models. The advantages of this setup over a single, fully integrated model are twofold: First, it is more flexible, leaving the constituent models intact for independent runs, thus making further model development an easier task. Second, the decomposed model solution benefits numerically from having the most non-linearities concentrated in the smaller of the two modules. The emphasis of the paper is on methodology, but we also include an example demonstrating the feedback mechanisms of MESSAGE-MACRO by applying it to two global economicenergy-environment scenarios. The two scenarios are a reference scenario and a scenario that limits the global atmospheric carbon concentration to $550 \mathrm{ppmv}$. The scenarios are compared in terms of GDP, energy supply and demand, and energy prices. (C) 2000 Elsevier Science Ltd. All rights reserved.
\end{abstract}

\section{Introduction}

This paper describes MESSAGE-MACRO, one part of the IIASA Integrated Assessment Scheme for Scenario Analysis. The paper emphasizes the description of the model, but illustrates its main features with typical results.

MESSAGE-MACRO is the result of linking an energy supply model of the Linear Programming type (MESSAGE) with a non-linear macroeconomic model (MACRO). The link is fully automated, but involves running the two parts in a stand-alone mode. In comparison with fully

\footnotetext{
* Corresponding author. Fax: +43-2236-71313.

E-mail address: leo@iiasa.ac.at (L. Schrattenholzer)
} 
integrated models such as MARKAL-MACRO [13], our approach lacks the elegance of a single black box but it has, perhaps, more transparency. We are also convinced that it is more versatile and faster than a fully integrated model. In any case, and under the given circumstances, it was the most straightforward tool to produce for the purpose.

The motivation for building this tool came from the desire to improve earlier "soft-linked" [17] versions of predecessor models. That soft linking involved overlapping domains of the linked models which led to inconsistencies between a part of the results that could never be reconciled completely.

\subsection{The IIASA integrated assessment scheme for scenario analysis}

IIASA's Environmentally Compatible Energy Strategies (ECS) project has developed an integrated set of tools, the IIASA Integrated Assessment Scenario Analysis Framework. A schematic overview of this computational framework, which shows the connections between its parts and the kind of information that flows between them, is given in Fig. 1.

One central part of this framework, MESSAGE-MACRO, describes the interaction between macroeconomic production, energy demand and supply, and pollutant emissions. The inputs into

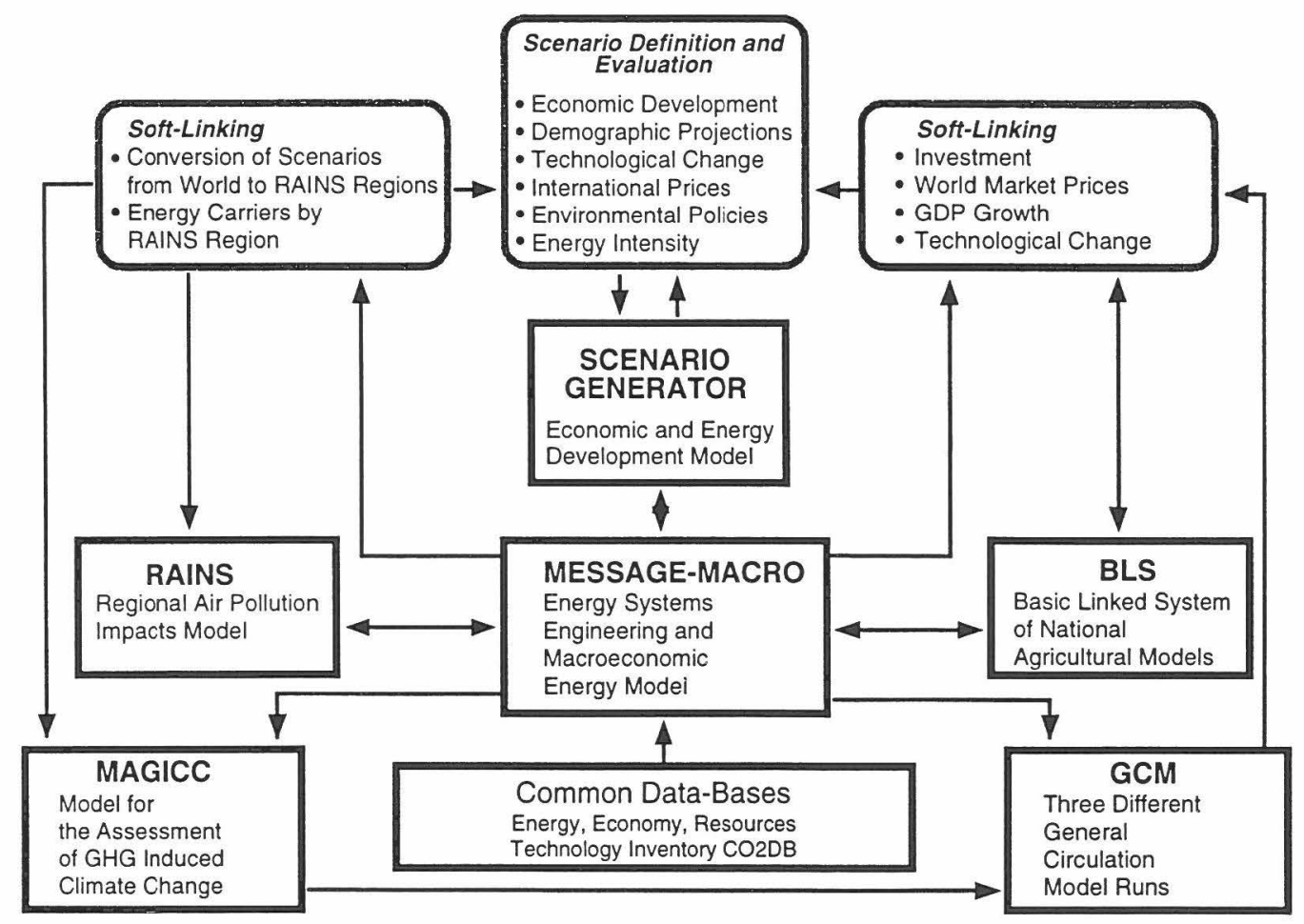

Fig. 1. The IIASA Integrated Assessment Scheme for Scenario Analysis. 
MESSAGE-MACRO, reference values of economic growth and of overall energy intensity of gross domestic product (GDP), come from the Scenario Generator, a database of historical economic development indicators and energy supply variables. The Scenario Generator generates these inputs by combining qualitative, highly aggregated scenario assumptions, e.g., on the speed of convergence of GDP per capita in the world regions over time and on technological change, with extrapolations of historical trends. ${ }^{1}$ Other inputs into MESSAGE-MACRO come from formal databases and other data sources such as publications in the field. A major source of present and future data on technologies is CO2DB, described in Strubegger et al. [16]. One internal database contains data on the availability of primary energy sources. The third major group of inputs into the energy supply part of MESSAGE-MACRO is energy demand. Energy demand is again derived from the Scenario Generator. More precisely, the Scenario Generator provides an initial trajectory of energy demand as a starting point for the iterative procedure to solve MESSAGEMACRO. In its final result, energy demand is endogenous in MESSAGE-MACRO and subject to macroeconomic optimization.

The outputs of MESSAGE-MACRO on pollutant emissions enter directly into the Regional Air Pollution Impacts model RAINS [1,3] and an advanced version of MAGICC [19,6]. The latter two models calculate environmental impacts (sulfur depositions, atmospheric $\mathrm{CO}_{2}$ concentrations, temperature averages, etc.), which are inputs into the Basic Linked System of National Agricultural Models, BLS [5]. BLS and MESSAGE-MACRO are also soft-linked; that is, they use consistent data on macroeconomic investments, GDP growth, and technological change.

This scheme of models and its operation reflects past model developments at IIASA and its modeling needs and practices. It is noted that this is different from integrated models which are much more closely linked and which usually include an explicit feedback from the climate to the economy. One prominent example of integrated models is IMAGE, developed at the Dutch RIVM [2]. For an overview and comparison of approaches and results of Integrated-Assessment modeling see Weyant et al. [18]. An example of a model that integrates all sectors of the economy and that includes, in addition to $\mathrm{CO}_{2}$ seven other greenhouse gases, is the Second Generation Model SGM developed at the US-PNL [4].

\subsection{The development of MESSAGE-MACRO}

Prior to the development of MESSAGE-MACRO and its inclusion in IIASA's Integrated Assessment Scheme, two separate models, MESSAGE and 11R, played the role that is now fulfilled by the linked modules. $11 \mathrm{R}$ is a model of energy-economy-environment interactions, building on the Global 2100 model by Manne and Richels [12]. In the earlier setup, both MESSAGE and $11 \mathrm{R}$ included a representation of the Reference Energy System (RES), MESSAGE's RES being much more detailed. This duplicity introduced variety and enriched the view of the future, which, given the time frame of more than 100 years, cannot reasonably be pictured unambiguously anyway. However, it was unsatisfactory that scenario variability was introduced by peculiarities of the respective energy models rather than by well-defined scenario variables.

In the course of time, the negative aspects of having separate models, perhaps aided by the

\footnotetext{
' For a detailed description of the Scenario Generator, see Gritsevskii [8].
} 
increased elegance of the result envisaged, weighed heavier than the advantages, leading to a linking of MESSAGE and $11 \mathrm{R}$. Readers familiar with energy-economy optimization models may know the MARKAL-MACRO model [13] which has much in common with our model. The main difference between the two approaches is that MARKAL-MACRO is a fully integrated single model, whereas MESSAGE-MACRO is solved by running each part separately and iterating their inputs until consistency between the macroeconomic part and the energy part is reached. In this solution process, the iterations have the function to match demand and supply curves in 11 world regions in all time periods.

The vehicle transporting the information between the two submodels are supply curves derived from MESSAGE. These supply curves are input to MACRO, which returns a new set of demands, consistent with MACRO's production function, to MESSAGE.

\section{Short model descriptions}

The following description of MESSAGE-MACRO is divided into the description of the two constituent models, followed by the description of the linking procedure. In our view, this division not only enhances clarity, it also reflects the fact that both models can still be run independently from each other.

\subsection{The systems engineering model MESSAGE III}

MESSAGE III is a dynamic systems engineering optimization model that is used for medium to long-term energy planning, energy policy analysis and scenario development [14]. The model provides a framework to represent an energy system with all its interrelations and dependencies. At the core of MESSAGE is a reference energy system (RES) that represents the most important energy carriers and conversion technologies.

The exact shape of the RES and its development over time (technological progress) is defined by the model input data. For the runs reported here, the RES included the fossil energy carriers coal, oil and gas, nuclear, and various renewable sources of energy. Final energy is available in the form of liquid, solid and gaseous fuels, electricity and district heat. Methanol and hydrogen can be introduced as an alternative to the presently available energy carriers.

Energy demands are exogenous to the model. They are defined on the level of useful energy, distinguishing between thermal and specific (mostly electricity) uses in the industry and residential/commercial sectors, and between passenger and freight transport demands.

The geographical disaggregation is into 11 world regions that are linked by international trade of primary energy carriers as well as the final energy carriers methanol and hydrogen. For each of the 11 regions the RES includes approximately 150 technologies.

MESSAGE results include optimal (i.e., least-cost) energy supply and utilization structures, resource extraction profiles, marginal cost and quantities of energy traded internationally, investment requirements in the energy sector, and pollutant emissions.

Generally, MESSAGE III can be applied to check the technical feasibility of a projected development of the energy system, i.e., that resources are sufficient, that technologies are adequate for the energy system, and that the developments over time, especially the demand trajectories, are consistent with the setup of the scenario. 
Energy supply responds to energy prices in MESSAGE in the form of substitution effects guided by the overall optimization procedure. Elastic demand in the sense of reducing the use of energy due to higher energy prices can be implemented using a step-wise approximation [14]. However, this feature of MESSAGE is not easy to calibrate or to handle, so linking the model to an economic model as described here seems a preferable solution.

\subsection{MACRO: a macroeconomic model of energy-economy interactions}

MACRO, as it is used in the link with MESSAGE, has its roots in a long series of models by Manne and others. The latest model in this series is MERGE-3 [11]. ${ }^{2}$ MACRO is a macroeconomic model maximizing the intertemporal utility function of a single representative producerconsumer in each world region. The optimization result is a sequence of optimal savings, investment, and consumption decisions. The main variables of the model are the capital stock, available labor, and energy inputs, which together determine the total output of an economy according to a nested CES (constant elasticity of substitution) production function. Energy demand in two categories (electricity and non-electric energy) is determined within the model, and is consistent with energy supply curves, which are inputs to the model.

The model's most important driving input variables are the projected growth rates of total labor, i.e., the combined effect of labor force and labor productivity growth and the annual rates of reference energy intensity reduction.

Labor growth is also referred to as reference GDP growth. In the absence of price changes, energy demands grow at rates that are the approximate result of GDP growth rates, reduced by the rates of overall energy intensity reduction. Price changes can alter this path significantly.

\section{The link}

\subsection{The cost function}

MACRO's production function includes two energy demand categories, electric and non-electric. To optimize, MACRO requires cost information for each demand category. The exact definitions of these costs as a function over all positive quantities of energy cannot be given in closed form-as formulae-because each point of the function would be a result of a full MESSAGE run. However, the optimality conditions implicit in the formulation of MACRO only require the functional values and its derivatives at the optimal point to be consistent between the two submodels. Since these requirements are therefore only local, most functions with this feature will simulate the combined energy-economic system in the neighborhood of the optimal point.

We have chosen a quadratic form, that is,

$$
\operatorname{cost}_{t}=c_{t}+\sum_{j} a_{j, t} \times \text { energy }_{j, t} \times \text { energy }_{j, t} ; \quad j=\text { ELEC,NELE; } t=1, T
$$

\footnotetext{
2 Licenses for MERGE-2 and MERGE-3 are available through http://www.stanford.edu/group/MERGE/.
} 
The parameters $c_{t}$ and $a_{j, l}$ were determined such that the resulting costs and their derivatives coincided with MESSAGE's outputs in each point in time and in both demand sectors. A schematic illustration of one component of the cost function (that is, for one demand sector and one time period) is depicted in Fig. 2. In the version of MESSAGE that has been used for the linking, seven demand sectors are defined. To accommodate the two energy sectors of MACRO, the seven sectors were aggregated into the two that are required.

\subsection{Optimality conditions}

To describe how the cost functions influence the optimization, we first present the production function of MESSAGE-MACRO.

$$
Y_{t}=\left(a \times K_{t}^{\rho \times \alpha} \times L_{t}^{\rho \times(1-\alpha)}+b \times E_{t}^{\rho \times \beta} \times N_{t}^{\rho \times(1-\beta)}\right)^{(1 / \rho)}
$$

with $Y_{t}$ output at time $t, K_{t}$ capital stock at time $t, L_{t}$ labor force at time $t, N$, non-electric energy demand at time $t, E_{t}$ demand for electricity at time $t, \alpha$ optimal share of capital in the capital-labor aggregate, $\beta$ optimal share of electricity in the energy aggregate, $\rho=(1-1 / \sigma)$ (with $\sigma=$ elasticity of substitution), $a, b$ calibration parameters.

This formulation of the production function implies that each production factor increases total production up to the point where its price begins to exceed (i.e., is equal to) the partial derivative of the production function with respect to this production factor. For the calibration year, this point (condition) then provides, together with the values of the variables in the calibration year, the two equations needed for the calculation of the two calibration parameters $a$ and $b .^{3}$ The calibrated production function, together with the cost curve, drives the optimization between MESSAGE and MACRO. This explains why it is only the local properties of the cost function that matter for the optimization.

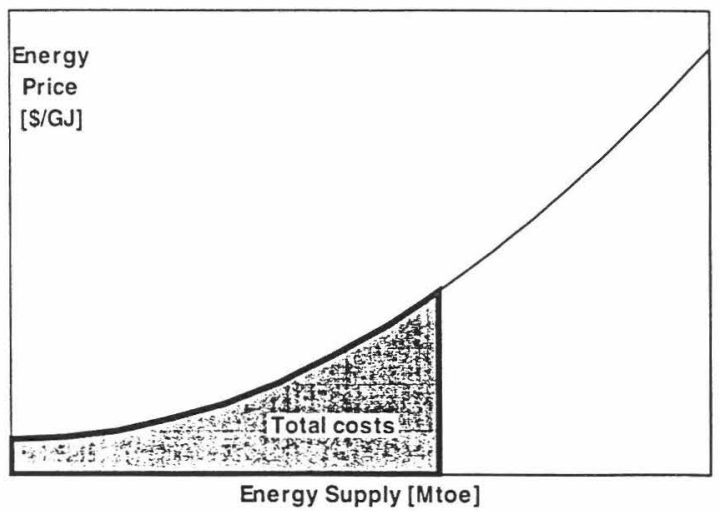

Fig. 2. A schematic presentation of energy supply costs in MESSAGE-MACRO.

\footnotetext{
${ }^{3}$ Any production factor could be used for the partial derivative and thus for the formulation of the second equation necessary to determine $a$ and $b$. In actual model use, the price of non-electric energy serves this purpose.
} 


\subsection{Iterating between MESSAGE and MACRO}

The algorithm to solve the linked MESSAGE-MACRO is a straightforward iteration, beginning with a MESSAGE solution. A schematic overview of the algorithm is presented in Fig. 3 . The dashed lines in the figure indicate one-time input flows. The uninterrupted lines describe the information flow during the iterations. The one-time inputs from the Scenario Generator are trajectories of final energy demand and of economic development. These two time series are converted to growth rates of potential GDP and to rates of energy intensity reduction. Since GDP and energy demand are subject to the optimization process in MESSAGE-MACRO, the inputs from the Scenario Generator cannot provide the eventual quantities, but only reference values needed by the optimization model as starting values. In practice and under "business-as-usual" conditions, solution values do not deviate much (typically not more than 5\%) from the reference values.

The final-energy demand from the Scenario Generator is also converted into useful-energy demand, which is needed by MESSAGE to drive its supply optimization. Again, initial demand is subject to subsequent change during the optimization process, but this step is necessary to establish an initial distribution of the two final-energy forms (electric and non-electric) in all demand sectors of MESSAGE.

After these one-time inputs have been made, the iteration proceeds independent of further inputs by the Scenario Generator. The first step of the iteration is a MESSAGE solution. Based on this solution, resulting demands are aggregated into the two sectors electric and non-electric. For the

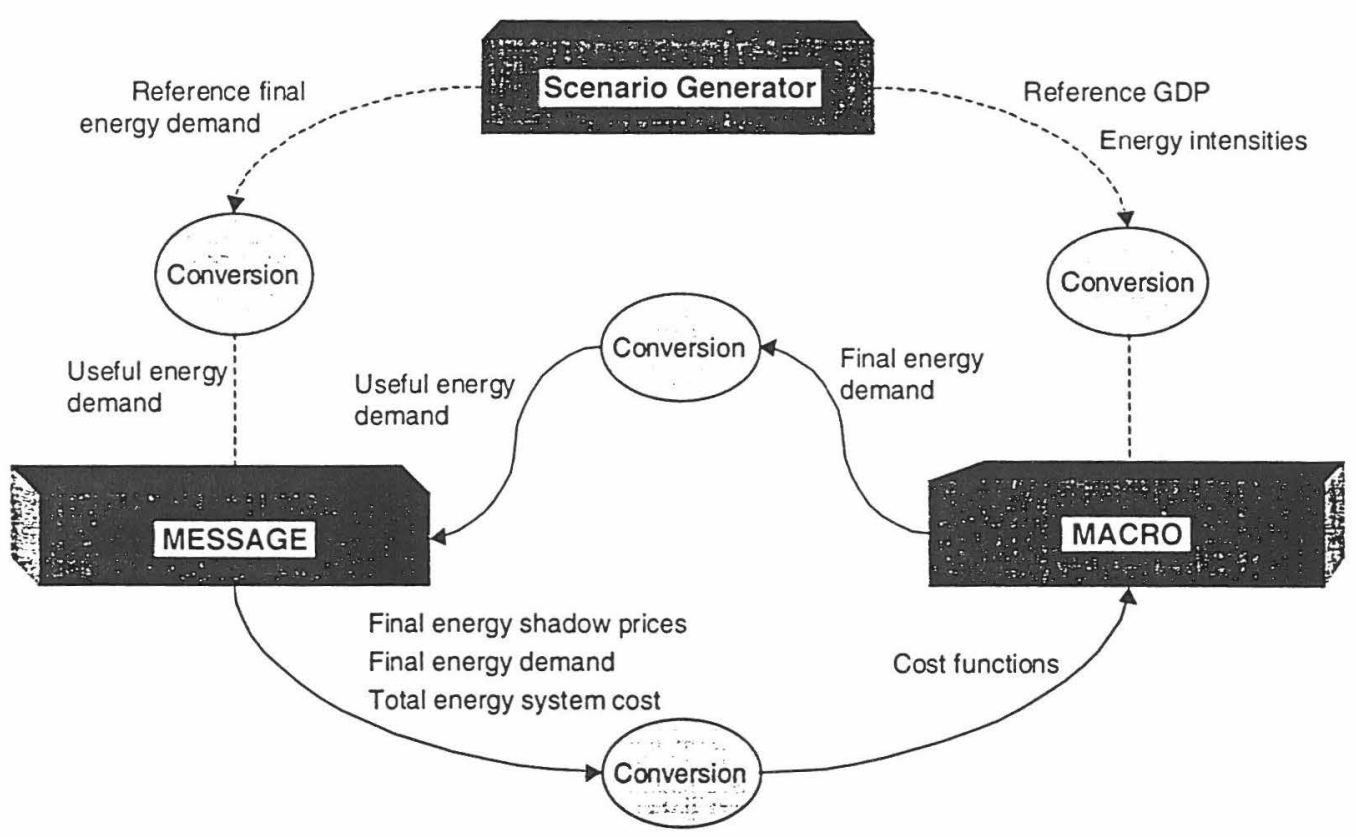

Fig. 3. Schematic overview of MESSAGE-MACRO iterations. 
same two categories, shadow prices are calculated as weighted averages. In addition, total energy systems costs are calculated. With this information, two cost functions-as defined above - are calculated for each future time period and each world region. For the base year, MACRO requires no cost functions, because all model parameters are fixed, and the information on prices and quantities is used to calibrate the production function parameters.

MACRO is then run with these inputs, and optimized demands are calculated. From these demands, correction factors (the ratios between new and old demand figures) are calculated and MESSAGE demands are updated. This completes the first step of the iteration. The iterations end as soon as the correction factors fulfill a convergence criterion. Thus far, we were satisfied with the speed of convergence of the algorithm. In the following section, we illustrate a typical convergence process.

\subsection{Software}

MESSAGE-MACRO runs under a UNIX operating system. The model-generating codes for both submodules are written using the $\mathrm{C}$ programming language. MACRO is formulated in GAMS, and the computer image of MESSAGE is a matrix in MPS format, which can be solved by several commercial solvers. The model corresponding to the documented runs has approximately 50,000 constraints. It was solved using HOPDM [7]. The linking is controlled by a UNIX shell script. The solution time required for one MESSAGE iteration is in the order of one hour, MACRO runs in less than five minutes.

\section{Results}

\subsection{Illustration of convergence}

Owing to the small fraction of economic output that is used to pay for the energy supply of an economy, adjustments during the iterative solution of MESSAGE-MACRO are relatively smaller in total economic output than they are in energy prices and quantities. The first part of this assertion is illustrated in Fig. 4, showing the movement of global GDP during the first five iterations of the MESSAGE-MACRO solution procedure.

Note that the total vertical range covered by this graph is two percentage points. The maximum deviation of any two GDP developments is thus well below $1 \%$, and from the third iteration onwards, there is a practical standstill of the model's GDP projection.

At the other end of the scale-in terms of the dynamics-is the evolvement of shadow prices during the iterations. Fig. 5 shows the paths of shadow prices of non-electric energy, averaged over the 11 world regions, during the five iterations. In contrast to the movement of GDP during the iterations, these shadow prices move over a much wider range. The biggest deviation between the first and the last iteration exceeded $10 \%$. Nonetheless, the convergence seems rather fast. ${ }^{4}$

\footnotetext{
+ Convergence might have been even faster if the correction factors-those correcting the demands transferred from MACRO to MESSAGE-would not be constrained in each iteration. This constraint was introduced into our experiments at an early stage to prevent extreme oscillations. Since the iterations work well with this constraint, there seems to be no reason for eliminating them Equally, their significance does not seem high enough to warrant a separate analysis of their optimal size.
} 


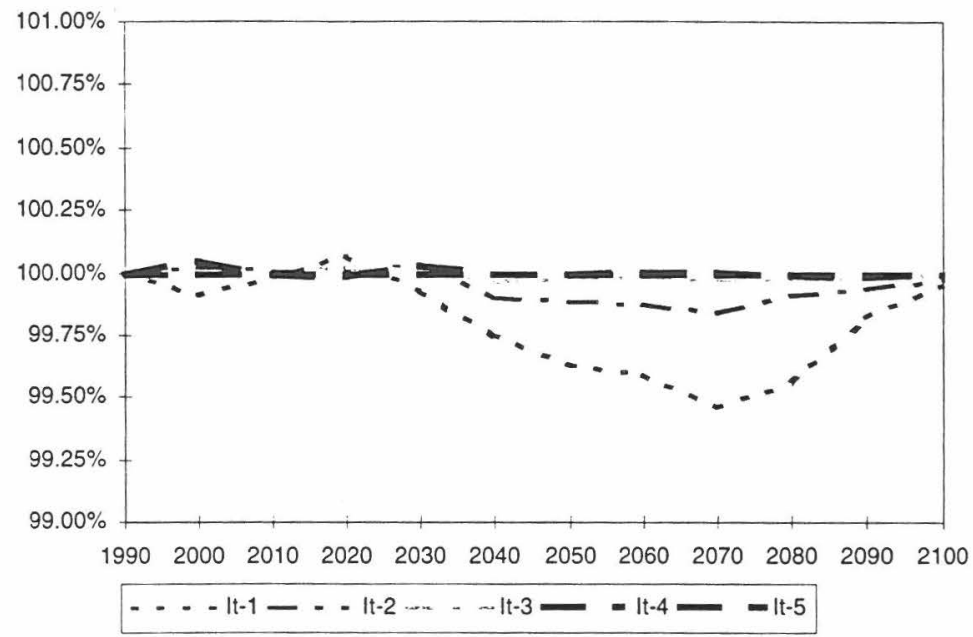

Fig. 4. Global GDP, five iterations in the solution of MESSAGE-MACRO. Units: percent of the fifth iteration.

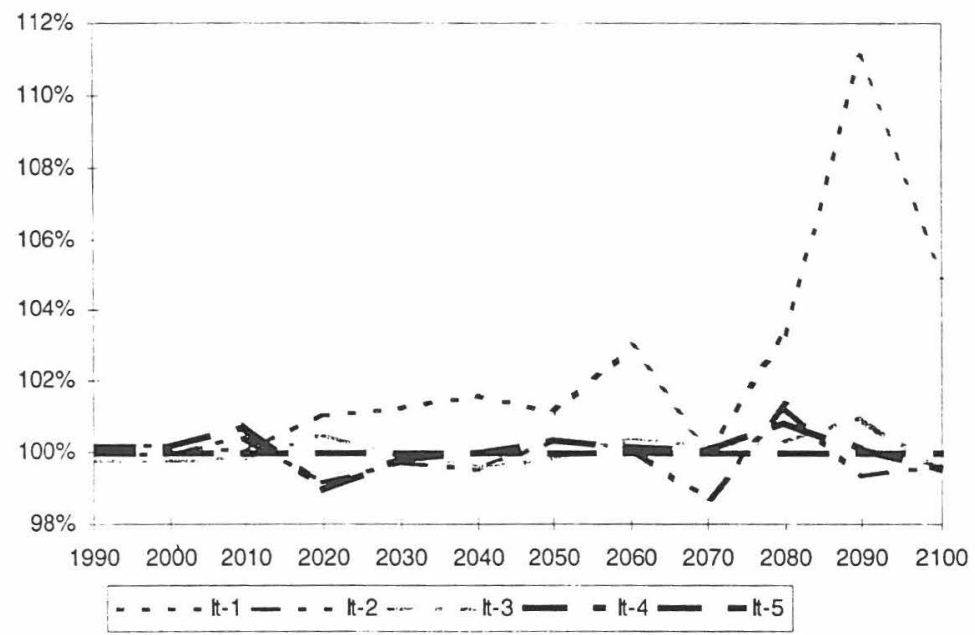

Fig. 5. Shadow prices of non-electric energy, five iterations in the solution of MESSAGE-MACRO. Units: percent of the fifth iteration.

To complete the illustration of convergence, we show, in Fig. 6, the development of global non-electric energy demand during the five iterations with MESSAGE-MACRO, again expressed as percent of the fifth iteration. As expected, the figure is almost a mirror image of the movements of the price curve shown in Fig. 5, because MACRO increases demands when prices are falling. The question remains, why, during the iterations, did MESSAGE detect the possibility to deliver 


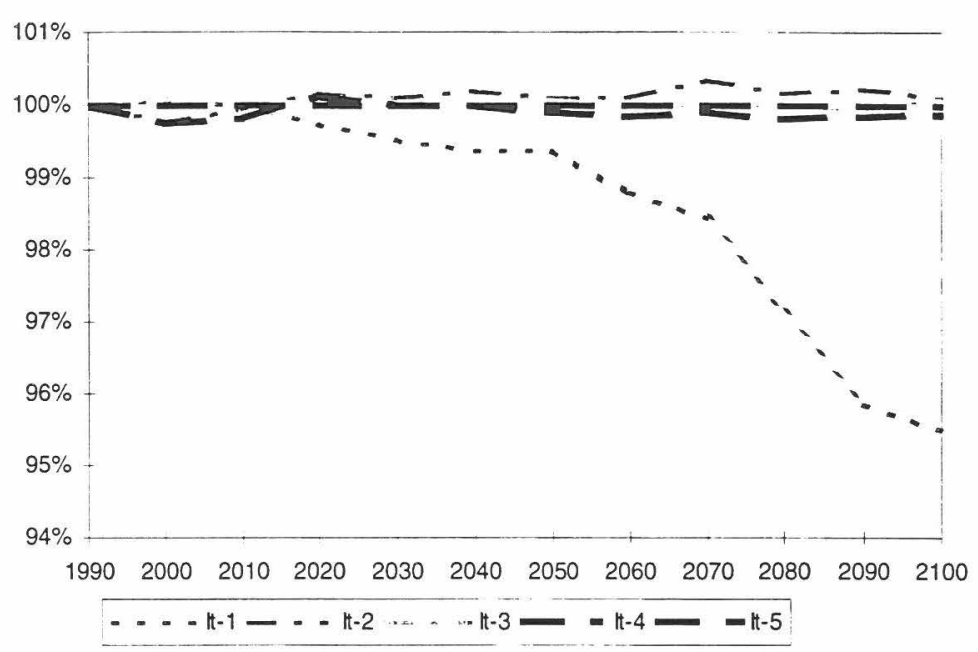

Fig. 6. Global non-electric energy demand; five iterations with MESSAGE-MACRO. Units: percent of the fifth iteration.

more non-electric energy at lower costs than initially? The reason is the interplay between electric and non-electric energy in the production function of MACRO, which allows substitution between these two final energy forms. We do not show the development of electricity demand in separate figures, but we just confirm that during the iterations, electricity demand decreased as demand for non-electric energy went up.

Technically, the substitution between electric and non-electric energy is influenced by a parameter of MACRO's production function that specifies the optimal value share (i.e., total cost) of electricity in the aggregate of electric and non-electric energy. In our scenarios, this parameter increases over time so as to reflect the relative increase of electricity demand within each economic sector. This parameter is therefore a so-called "scenario variable", chosen in a way to mimic historical trends.

\subsection{Two scenarios generated with the linked model}

Achieving internal consistency between the different design elements of a scenario as expressed by the Scenario Generator is the goal of a single run of MESSAGE-MACRO. To achieve this consistency is the purpose of the iterations illustrated in the previous section. At the end of this iteration a scenario exists that is consistent with the Scenario Generator's assumptions about economic growth and technological progress, with MACRO's description of the economic machinery that produces output (i.e., the production function), and with MESSAGE's representation of the detailed RES.

The internal functioning of MESSAGE-MACRO that leads to this consistency may be considered a worthwhile feature, but an even more important kind of consistency comes into play when we proceed from one scenario to another. In principle, the issues are the same, i.e., to 
combine bottom-up effects of providing an optimized energy supply system with top-down effects concerning the substitution between the different production factors as a consequence of price changes.

To illustrate a case of scenario comparison, we present, in the following subsection a global E3 (energy-economy-environment) reference case together with a greenhouse gas mitigation scenario. The latter is defined by imposing carbon emission limits on MESSAGE. The case without such limits is the reference case with which the results of the mitigation case are compared.

As in the previous section, the prime purpose of the description here is to illustrate the functioning of MESSAGE-MACRO and not to present a result that is of direct policy relevance. To provide a minimum of background information, however, we will describe the scenarios to the extent that the results illustrate the method used, but we ask the readers to bear with us if we restrict the arguments in support of specific assumptions and input data to a minimum. Readers interested in the substantive part of the scenarios are referred to IIASA-WEC [10] for more detail.

\subsection{Two global energy-economy-enviromment scenarios}

To emphasize the illustration of the method, the two scenarios described here were chosen in part so as to create a major "disturbance" to be inflicted on a reference scenario. The reference scenario is therefore one with particularly high use of coal as a primary energy source. In this scenario, global energy-related $\mathrm{CO}_{2}$ emissions ${ }^{5}$ increase beyond 24 billion tons of carbon $(\mathrm{GtC})$ i.e., approximately four times 1990 values-by the end of the next century. In a second scenario, Carbon Mitigation Case, total energy-related $\mathrm{CO}_{2}$ emissions are restricted, in MESSAGE, so as to keep atmospheric concentration of this greenhouse gas below 550 ppmv. This level means approximately twice pre-industrial concentrations and is a kind of "anchor" value used in many studies of the climate problem. Note that this specific formulation of a greenhouse gas emission constraint permits the model to choose an optimal time path for costly emission reductions. Accordingly, emission reductions are postponed until a point in time in which discounting - both of costs in MESSAGE and of utility of consumption in MACRO - reduces the effect of mitigation costs on the objective functions. This effect limits the climate-related objectives represented by the mitigation scenario to achieving an asymptotic goal in a cost-effective way. No attempt is made to balance costs and benefits of mitigation. The benefits may well depend on the shape of the emission path leading to the eventual limit [15]. If, for instance, the rate of the increase of the atmospheric concentration of greenhouse gases is critical-and therefore constrained in addition to its absolute limit-mitigation must occur faster and, therefore, incurs higher costs. (For a discussion of the consequence of timing on mitigation strategies, see, e.g., Grübler and Messner [9]).

In MESSAGE-MACRO, the cost of emission reduction is the result of two effects. One is the direct effect of a higher share of total economic output that goes into the procurement of cleaner energy. This effect is partly offset by the second effect, the benefits resulting from the model's ability to substitute capital for energy. Direct and the indirect effects together then result in a lower GDP in the Mitigation Case.

\footnotetext{
5 In our scenarios. these are defined as $\mathrm{CO}_{2}$ emissions resulting from the combustion of fossil fuels plus emissions from the use of fossil primary energy as feedstock material.
} 
Summarizing the main results of the two scenarios in one graph, Fig. 7 shows the energyrelated $\mathrm{CO}_{2}$ emissions in the two scenarios and the concomitant GDP losses relative to the Reference Case. The differences between the two emission paths and the GDP reductions begin to be noticeable after the year 2010. By the year 2100, emission reductions increase to $80 \%$ of the Reference Case. GDP losses exceed $0.5 \%$ from the year 2050 onwards. They remain between 1.5 and $1.7 \%$ during the last three periods of the model's time horizon.

To illustrate the effect of substitution of energy by capital, Fig. 8 shows the trajectories of total primary energy use over time in the two scenarios. By the year 2100 , total final energy demand in the Mitigation Case is reduced by some $15 \%$ relative to the Reference Case.

This substitution is induced by the increase in marginal final-energy costs due to expenditures for carbon mitigation measures. By 2100 marginal costs of non-electric energy, as shown in Fig. 9, are $77 \%$ higher in the Mitigation Case than in the Reference Case (as an average over the 11 world regions). This difference in marginal costs exceeds 5\% in 2040, when carbon emissions in the Mitigation Case begin to be lower by more than $10 \%$ than those in the Reference Case.

Changes in electricity costs are, with a $37 \%$ increase, approximately half of the increase of non-electric energy prices. This is due to the higher flexibility in electricity generation, where various power plants using different energy sources can be used to produce the same final-energy carrier. This fact is also reflected in the relative changes in electric and non-electric energy requirements of the two cases: non-electric energy consumption is reduced by $20 \%$ in 2100 , while for electricity use the reduction is only $6 \%$.

Fig. 10 gives an indication of how the reduction in carbon emissions in the Mitigation Case is reached. Compared to the Reference Case, overall primary energy consumption is reduced by $10 \%$, with a decrease in coal consumption by $70 \%$. Gas consumption remains unchanged, while the use of oil increases slightly. Carbon-free energy supply options like solar PV cells and nuclear reactors account for more than two thirds of primary energy.

Marginal costs of mitigating carbon emissions, as derived by the optimization of the energy

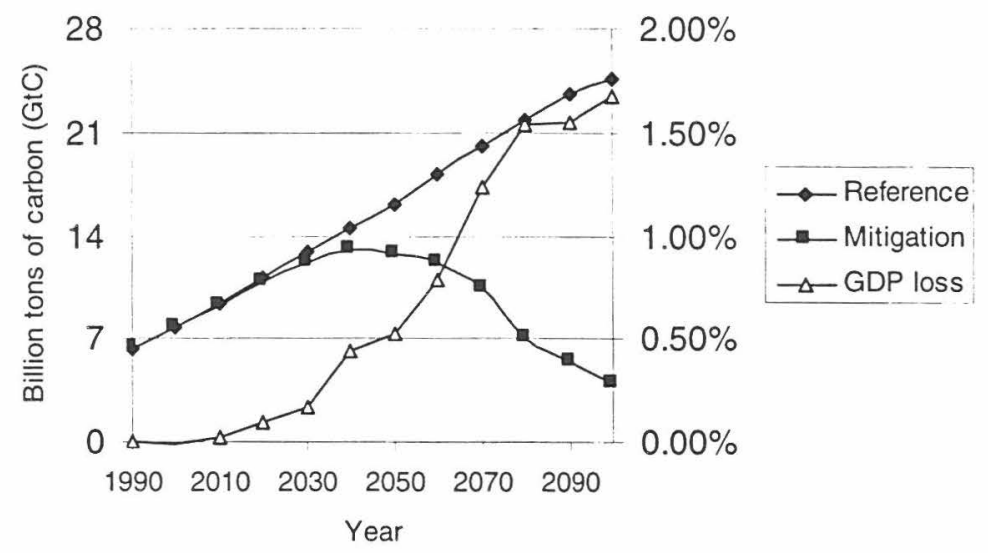

Fig. 7. Carbon emission paths in the Reference Case and in the Carbon Mitigation Case; GDP losses in the Mitigation Case relative to the Reference Case. 


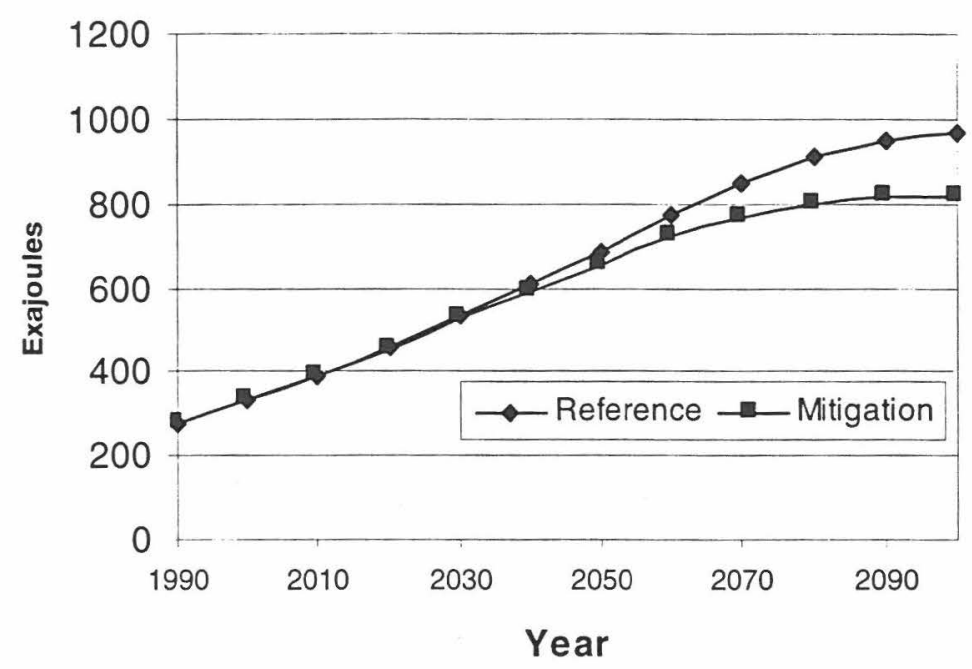

Fig. 8. Total final energy demand in two scenarios, exajoules.
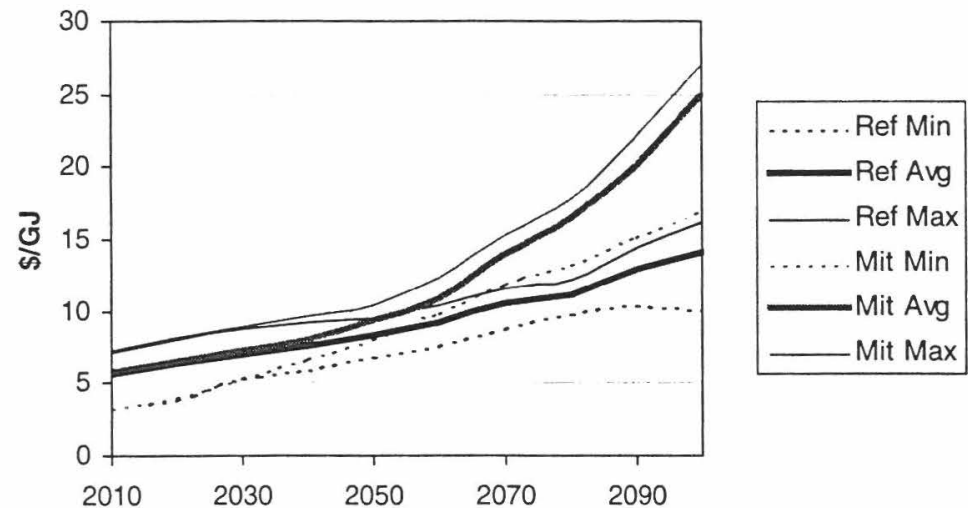

Fig. 9. Marginal costs of non-electric final energy in the Reference and Mitigation Cases (average, minimum and maximum over the regions in $\$ / G J)$.

supply system with a cumulative carbon emission constraint is around US\$3 per ton C as net present value in 1990. For the year 2100 and given the discount rate of $5 \%$ used, this amounts to US\$711 per ton C.

\section{Discussion}

In the linked MESSAGE-MACRO model, the consistency between economic output and energy supply costs means that higher energy prices as a consequence of meeting carbon emission limits 


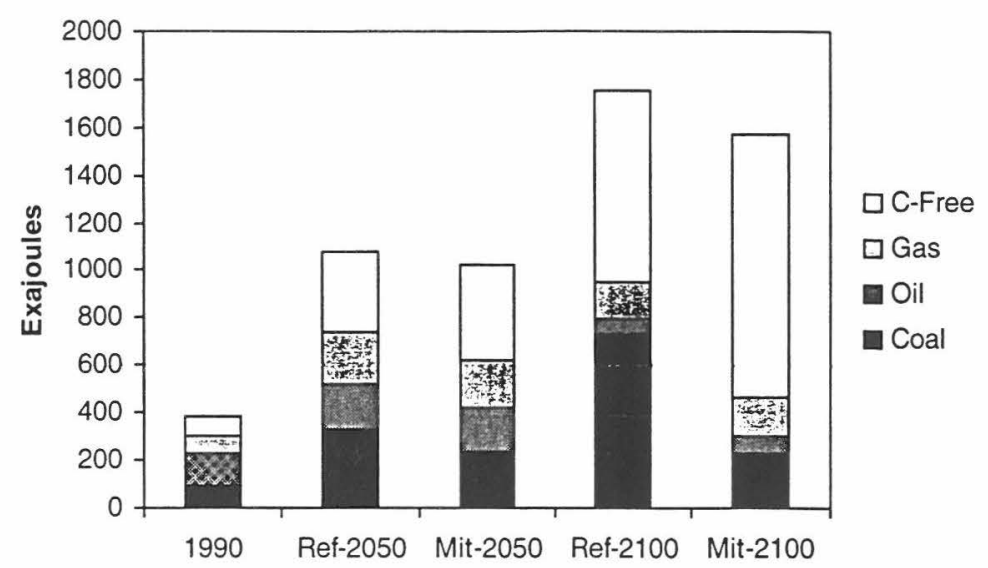

Fig. 10. Composition of primary energy supply in 1990, 2050, and 2100 for the Reference and the Mitigation Case, exajoules.

lead to a substitution of the production factor energy by capital and labor. Since this substitution is not completely elastic, adjustment costs are incurred, which reduce GDP relative to the reference case. At the same time, however, the substitution means that GDP loss is less than simply the cost differences of reference and mitigated energy supply.

Another example of consistency between scenarios that could be quantified with MESSAGEMACRO, not illustrated here, would be the illustration of the so-called "rebound" effect. This effect describes increases of energy demand as a consequence of increased efficiencies of energy conversion-and thereby decreased final-energy costs.

Expressed in general terms, through the consistency between scenarios, MESSAGE-MACRO addresses potential weaknesses of pure "Top-down" and pure "Bottom-up" modeling approaches. Potential weaknesses of Top-down models include an underestimate of technological progress, which is described here not only by exogenous inputs on energy intensity reduction in the MACRO part but also by parameters describing improving technological performance in MESSAGE. On the other hand, Bottom-up models can easily fail to catch the take-back effect, which is captured here by the MACRO part of the linked model.

Up to this point we have discussed the methodological aspects of linking the two models as described. We hope to have thereby contributed to the discussion of long-term energy modeling, in particular of Bottom-up vs Top-down approaches. That is a vast field, and our contribution has a limited scope by design. Just to establish a connection to the full subject, we want to discuss some of the inherent features of the models used, in particular those that are considered problematic by some.

To begin with, some would question the suitability of macroeconomic models for the longterm analysis of economic development. Their argument is that any production function describes only one production system, which is subject to change in response to changes such as oil-price hikes and an impending climate catastrophe. There are several answers to this concern. The first is that suitability is a question of degree and there is no tool that is fully suitable to model the 
global economy over the next 100 years. Readers must judge themselves whether the outputs of our model makes sense to them, but we address mainly those readers who accept the methods used as contributing important aspects to the analysis of long-term systems analysis. The next answer to the question about the appropriateness of our production function is that it does respond to changes such as price increases as a result of resource scarcity or environmental constraints. Moreover, our production function is re-calibrated for each time period to describe technological progress over and above the overall reduction of energy intensity. (We did not go into this much detail of the model description because we wanted to focus the presentation on the linking.)

As for all models, the quality of the results is determined to an overwhelming extent by the quality of the input data. This is of particular importance if policy-relevant results are to be derived from MESSAGE-MACRO runs. Readers who want to form their own judgement about the quality of the data used for our scenarios are referred to a comprehensive description of the IIASA-WEC scenarios in IIASA-WEC [10]. Although the Reference Case presented in this report is not identical to any of the six IIASA-WEC scenarios, the most important data are.

\section{Summary}

This report describes how we have linked a macroeconomic and an energy supply model. The aim of the linkage was to build a tool that generates energy-economy-environment (E3) scenarios that are consistent in two respects. One is consistency between energy demand and energy supply cost curves owing to a price-responsive macroeconomic production function, and the other is consistency between two scenarios that describe the same economy but where one scenario includes additional constraints on its energy system. We consider this second kind of consistency more important than the first, and we have illustrated the response of the linked model to a constraint limiting global energy-related carbon emissions.

The emphasis of this paper was on methodology and not on policy analysis. In this spirit, the model results must be considered experimental, reported mainly to illustrate the reaction of the linked model to input variations. The results presented in this paper show that the chosen approach can be easily applied even for a large-scale model like the 11 region MESSAGE model used here. Furthermore a carbon Mitigation Case could be derived from a Reference Case by simply introducing a cumulative carbon constraint in the energy part. Effects on the energy system and the overall economy, including loss of GDP and reductions in energy consumption were derived from the analysis.

For the linking, we used a method that leaves the independence of the two constituent models, MESSAGE and MACRO, intact. This may seem unnecessarily "hand-woven", but it has the advantage of high flexibility. In MESSAGE-MACRO, it is easy, for example, to experiment with alternative production functions without carrying the burden of solving a big energy supply model each time. Our way of linking also has the advantage of collecting the non-linearities of the model in one place. This way, the non-linear model can be kept small and be solved in a short period of time. 


\section{Acknowledgements}

The authors wish to thank two anonymous reviewers as well as Profs. Alan S. Manne and Clas-Otto Wene for their helpful comments.

\section{References}

[1] Alcumo J, Shaw R, Hordijk L, editors. The RAINS model of acidification: science and strategies in Europe. Dordrecht: Kluwer Academic Publishers, 1990.

[2] Alcamo J, Battjes C, Van den Born GJ, Bouwman AF, de Haan BJ, Klein Goldewijk K, Klepper O, Kreileman GJJ, Krol M, Leemans R, van Minnen JG, Olivier JGJ, de Vries HJM, Toet AMC, van den Wijngaart RA, van der Woerd HJ, Zuidema G. Overview of IMAGE 2.0: an integrated model of climate change and the global environment. In: Zwerver S, van Rompaey RSAR, Kok MTJ, Berk MM, editors. Climate change research: evaluation and policy implications. Amsterdam: Elsevier Science. pp. 1395-1399.

[3] Amann M. Options, Winter '93. Laxenburg, Austria: International Institute for Applied Systems Analysis, 1993.

[4] Edmonds JA, Pitcher HM. Barns D, Baron R, Wise MA. Modelling future greenhouse gas emissions: the second generation model description. In: Klein LR, Lo F, editors. Modelling global change. United Nations University Press, 1995:295-340.

[5] Fischer G, Frohberg K, Keyzer MA, Parikh KS. Linked national models: a tool for international policy analysis. Dordrecht: Kluwer Academic Publishers, 1988.

[6] Fujii Y. A Reduced Model of the Global Carbon Cycle and Climate Change. Internal Paper. Laxenburg, Austria: International Institute for Applied Systems Analysis, 1996.

[7] Gondzio J, Makowski M. HOPDM, Modular solver for LP problems, User's guide to Version 2.12. WP-95-69. Laxenburg, Austria: International Institute for Applied Systems Analysis, 1995

[8] Gritsevskii A. Scenario generator. Internal Report. Laxenburg, Austria: International Institute for Applied Systems Analysis, 1996.

[9] Grübler A, Messner S. Technological change and the timing of mitigation measures. Energy Economics 1988;20(5):495-512.

[10] Nakicenovic N, Grübler A. McDonald A, editors. IIASA-WEC (World Energy Council) Global energy perspectives. UK: Cambridge University Press, 1998. See also http://www.iiasa.ac.at/Research/

[11] Manne A, Mendelsohn R, Richels R. MERGE-A Model for evaluating regional and global effects of GHG reduction policies. Energy Policy 1995;23(1):17-34.

[12] Manne A. Richels R. Buying greenhouse insurance: the economic costs of $\mathrm{CO}_{2}$ emission limits. Cambridge (USA): MIT Press, 1992.

[13] Manne A, Wene C-O. MARKAL-MACRO: A linked model for energy-economy analysis. In: Hake J-FR, Kleemann M. Kuckshinrichs W, Martinsen D, Walbeck M, editors. Advances in systems analysis: modelling energyrelated emissions on a national and global level. Jülich, Germany: Forschungszentrum Jülich, 1994:153-90.

[14] Messner S, Strubegger M. User's guide for MESSAGE III. WP-95-69. Laxenburg, Austria: International Institute for Applied Systems Analysis, 1995.

[15] Schneider SH. Integrated assessment modeling of global climate change: Transparent rational tool for policy making or opaque screen hiding value-laden assumptions? Environmental Modeling and Assessment 1997:2(4):229-50.

[16] Strubegger M, McDonald A, Gritsevskii A, Schrattenholzer L. CO2DB Manual, Version 2.0. Laxenburg, Austria: International Institute for Applied Systems Analysis, 1999.

[17] Wene C-O. Energy-economy analysis: linking the macroeconomic and systems-engineering approaches. WP-9542. Laxenburg, Austria: International Institute for Applied Systems Analysis, 1995.

[18] Weyant J., Convening Lead Author. Integrated Assessment of Climate Change: An overview and comparison of approaches and results. In: Bruce JP, Lee H Haites EF, editors. Climate change 1995: economic and social dimensions of climate change. Cambridge, UK: Cambridge University Press. pp. 367-396.

[19] Wigley TML. Salmon M, Raper SCB. Model for the assessment of greenhouse-gas induced climate change, Version 1.2. University of East Anglia (UK): Climate Research Unit, 1994. 